\title{
Optimizing Soft X-ray Spectromicroscopy for Fuel Cell Studies: X-ray Damage of Ionomer.
}

\author{
Lis G.A. Melo and Adam P. Hitchcock ${ }^{*}$ \\ Dept. of Chemistry and Chemical Biology, McMaster University, Hamilton, Canada \\ * Corresponding author, aph@mcmaster.ca
}

Polymer electrolyte membrane fuel cells (PEM-FC) using $\mathrm{H}_{2}$ as fuel are entering commercial use for personal automobiles, as a means to reduce greenhouse gas emissions while retaining performance and refueling times similar to ICE-based engines. Further optimization of PEM-FC focuses on reducing the amount of expensive catalyst and increasing reliability. Both factors depend on optimization of the distribution of components in the cathode catalyst layer (CL), particularly the perfluorosulfonic acid (PFSA) proton conduction ionomer. Soft X-ray Scanning Transmission X-ray Microscopy (STXM) at the $\mathrm{C}$ 1s and $\mathrm{F} 1 \mathrm{~s}$ edges has been shown to be a powerful tool to quantitatively map ionomer in the $\mathrm{CL}$ relative to carbon support, Pt catalyst and pores, in both 2D [1] and 3D [2]. However, PFSA is readily damaged. Achieving a quantitative understanding of the damage kinetics and mechanisms is of interest to design STXM analysis protocols that achieve maximum quality with acceptable levels of radiation damage. Accurate, quantitative reference spectra for various forms of PFSA (ionomer and membrane) have been measured [3]. The accuracy of thickness determination by X-ray absorption has been verified though a comparison to film thicknesses determined by UV-spectral reflectance [4]. This paper reports results of soft X-ray damage of PFSA spun cast thin films (50-200 nm) using several irradiation energies, and spectroscopy studies to determine quantitative critical doses and damage mechanisms.

The films were exposed in STXM to X-rays in $3 \times 3$ arrays of pads each with $10 \times 10$ pixels, with 60,120 and $180 \mathrm{~nm}$ pixel separation, at variable dwell times. The irradiation beam was defocused to the pixel spacing in each case. The absorbed dose (D, in MGy) was calculated from eqn. 1 [5], where $E$ is energy $(\mathrm{eV}), I o$ is incident flux (MHz), $t$ is dwell time (s), $s$ is the beam diameter at the sample (nm), $\rho$ is density in $\mathrm{g} / \mathrm{cm}^{3}, O D(E)$ is optical density of the sample at $\mathrm{E}, O D l(E)$ is optical density per nm of the material at $E(\mathrm{OD} / \mathrm{OD} 1$ is the sample thickness), and $\mathrm{K}$ is the measured detector efficiency $(0.4,0.6,0.8$ for $\mathrm{C}, \mathrm{O}, \mathrm{F}$ edges). This equation is a simplified form, which ignores sample thinning due to mass loss since the changes in dose due to reduced OD and reduced sample thickness roughly cancel [5]. When evaluating dose-damage relationships, first order kinetics is assumed and different damage phenomena are characterized by a critical dose, $a_{c}$, - the dose which decreases (or increases) a spectral feature by $1 / \mathrm{e}$. Damage analysis was performed at specific photon energies sensitive to specific damage channels.

$$
D=\frac{6.4 x 10^{5} \cdot E \cdot O D 1(E) \cdot I_{o}\left(1-e^{-O D(E)}\right) \cdot t}{K \cdot \pi \cdot s^{2} \cdot \rho \cdot O D(E)}
$$

Figure 1 plots the $\mathrm{C} 1 \mathrm{~s}, \mathrm{O} 1 \mathrm{~s}$ and $\mathrm{F} 1 \mathrm{~s}$ spectra of a $200 \mathrm{~nm}$ PFSA film as a function of dose. After only 20 MGy, there are visible changes. The two major $1 \mathrm{~s} \rightarrow \sigma^{*}(\mathrm{C}-\mathrm{F})$ peaks in the $\mathrm{C} 1 \mathrm{~s}$ and $\mathrm{F} 1 \mathrm{~s}$ spectra lose intensity; the $\mathrm{F} 1 \mathrm{~s}$ continuum intensity declines, and the broad $\mathrm{O} 1 \mathrm{~s}$ peak at $535-540 \mathrm{eV}$ related to the sidechain sulfonate and ether bonds [3], reduces intensity. At doses of $190 \mathrm{MGy}$, the two $\sigma^{*}(\mathrm{C}-\mathrm{F})$ peaks coalesce into one centred at $295 \mathrm{eV}(\mathrm{C} 1 \mathrm{~s})$ and $692 \mathrm{eV}(\mathrm{F} 1 \mathrm{~s})$, and new $\mathrm{C}=\mathrm{C}$ bonds (C 1s $\rightarrow \pi^{*}$ peak at $285 \mathrm{eV}$ ) and $\mathrm{C}=\mathrm{O}$ bonds (peaks at 287 and $532 \mathrm{eV}$ ) are formed. Further dosing induces only physical 
damage (decreased film thickness). Loss of fluorine is evidenced by reduction of $\mathrm{F}$ 1s signal and decrease in the signal below $284 \mathrm{eV}$ where $\mathrm{F} 1$ s valence absorption dominates.

Figure 2 plots the critical dose, $\mathrm{a}_{\mathrm{c}}$, of PFSA at 3 photon energies. The influence of the incident photon energy was evaluated by irradiating at 320 and $710 \mathrm{eV}$, while the influence of $\mathrm{O}_{2}$ was evaluated by comparing the $\mathrm{a}_{\mathrm{c}}$ measured in $\mathrm{He}$ with some residual $\mathrm{O}_{2}$, to that measured in vacuum (active pumping at $2 \times 10^{-4}$ mbar, negligible $\left.\mathrm{O}_{2}\right)$. Energies of $292.4,540$ and $690 \mathrm{eV}$ are $\mathrm{C} 1 \mathrm{~s} \rightarrow \sigma^{*}{ }_{\mathrm{C}-\mathrm{F}}\left(\mathrm{CF}_{2}\right), \mathrm{O} 1 \mathrm{~s} \rightarrow \sigma^{*} \mathrm{C}-\mathrm{O}(\mathrm{C}-$ $\mathrm{O}-\mathrm{C})$ and $\mathrm{F} 1 \mathrm{~s} \rightarrow \sigma^{*}{ }_{\mathrm{C}-\mathrm{F}}\left(\mathrm{CF}_{2}\right)$ transitions, respectively [3]. Damaging the film at $710 \mathrm{eV}$ leads to slightly higher $\mathrm{a}_{\mathrm{c}}$ at all 3 energies compared to damage at $320 \mathrm{eV}$ in He. The $\mathrm{a}_{\mathrm{c}}$ is expected to be similar at 292.4 and $690 \mathrm{eV}$ considering the transitions are related to the same bonds. The $\mathrm{a}_{\mathrm{c}}$ for breaking the ether bond $(540 \mathrm{eV})$ is smaller than $\mathrm{a}_{\mathrm{c}}$ for breaking C-F bonds, consistent with a larger sensitivity of the side-chain ether and sulfonate groups to damage, which was also found in membrane degradation studies mimicking fuel cell degradation conditions [6]. Operating with a smaller residual $\mathrm{O}_{2}$ concentration lowers the $\mathrm{a}_{\mathrm{c}}$ at 292.4 and $690 \mathrm{eV}$ to a similar value, which indicates some of the damage processes are catalysed by photooxidation [4,7]. Based on these studies, we recommend that quantitative STXM analytical methods for ionomer mapping be carried out in the strict absence of $\mathrm{O}_{2}$, with less than $20 \mathrm{MGy}$ total dose [8].

\section{References:}

[1] A. Putz et al, ECS Transactions 75 (2016) 3.

[2] J. Wu et al, J. Power Sources 381 (2018) 72.

[3] Z.B. Yan et al, J. Phys. Chem. C 122 (2018) 3233.

[4] L.G.A. Melo et al, Phys. Chem. Chem. Phys. (2018) in press, doi: 10.1039/C7CP08621K

[5] V. Berejnov et al, J. Syn. Rad. 25 (2018) 833-847.

[6] L. Ghassemzadeh et al., J. Phys. Chem. C 114 (2010) 14635.

[7] T. Coffey et al., J. El. Spec. Rel. Phen. 122 (2002) 65.

[8] Research was performed using the ambient-STXM at the Canadian Light Source, funded by CFI, and the polymer-STXM at the Advanced Light Source, funded by DoE BES. Research supported by AFCC, NSERC and the Catalyst Research for Polymer Electrolyte Fuel Cells network.

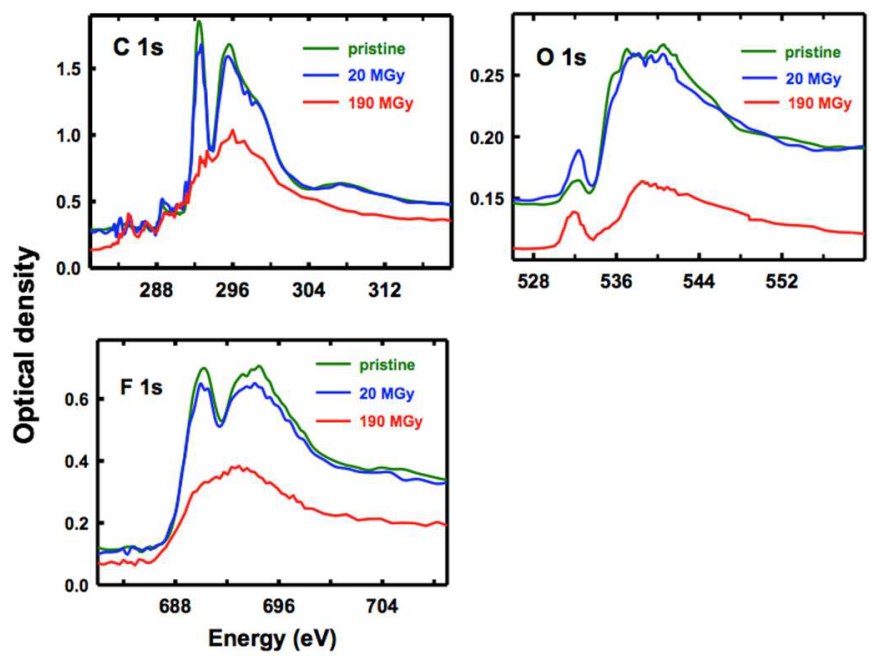

Fig. 1. $\mathrm{C} 1 \mathrm{~s}, \mathrm{O} 1 \mathrm{~s}$ and $\mathrm{F} 1 \mathrm{~s}$ spectra of a pristine 200 nm PFSA (<7 MGy dose) and after exposure at 320 $\mathrm{eV}$ to a low dose (20 MGy) and a high dose (190 MGy).

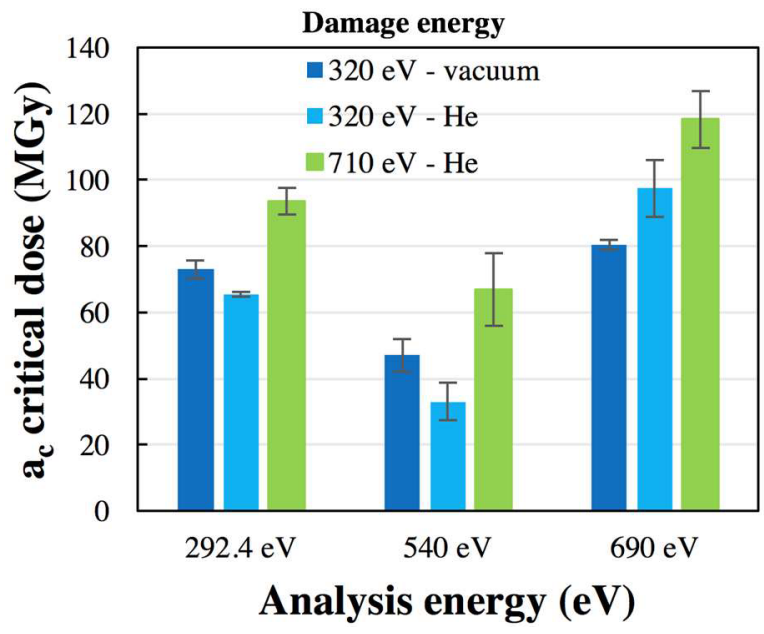

Fig. 2. Critical dose (MGy) of PFSA damaged at $320 \mathrm{eV}$ in vacuum, and 320 and $710 \mathrm{eV}$ under He, derived from an exponential fit to dose-damage data recorded at 292.4 and $690 \mathrm{eV}$ (C-F bonds), $540 \mathrm{eV}$ (sulfonate $\&$ ether bonds) 